\title{
Alimentação e ecomorfologia de duas espécies de piranhas (Characiformes: Characidae) do lago de Viana, estado do Maranhão, Brasil.
}

\author{
Nivaldo Magalhães PIORSKI ${ }^{1}$, José de Ribamar Lima ALVES, Monica Rejany Barros MACHADO, Maria \\ Marlucia Ferreira CORREIA
}

\begin{abstract}
RESUMO
Serrasalmus aff. brandtii e Pygocentrus nattereri são duas espécies de piranhas comuns no Lago de Viana, um lago formado a partir das inundações do Rio Pindaré, tributário da margem esquerda do Rio Mearim. Uma amostra composta por 249 exemplares destas espécies foi estudada a fim de identificar a composição da dieta e as estratégias alimentares empregadas pelas duas. Os peixes foram coletados trimestralmente durante um ano, entre março de 1998 e março de 1999, usando redes de emalhar e tarrafas. A composição qualitativa da dieta foi analisada com uso do método de freqüência de ocorrência. As estratégias alimentares empregadas pelas espécies foram inferidas através do método gráfico de Costello (1990), modificado por Amundsen et al. (1996). Os resultados indicaram que peixes foi o item encontrado com maior freqüência nos estômagos de ambas as espécies, seguido de matéria vegetal, com maior participação na dieta de P. nattereri. Os recursos peixes e vegetais tenderam a apresentar relações inversas quando comparados com as diferentes classes de comprimento. A análise gráfica da dieta de $P$. nattereri $S$. aff. brandtii sugere que a maioria dos indivíduos utiliza vários recursos simultaneamente. Uma análise multivariada de índices ecomorfológicos indicou que as espécies são segregadas com relação à habilidade natatória, posição ocupada na coluna d'água e tamanho relativo das presas.
\end{abstract}

PALAVRAS-CHAVE

Estratégia alimentar, Serrasalminae, ecomorfologia, água doce tropical.

\section{Feeding and ecomorphology of two species of piranbas (Characiformes: Characidae) from the Viana Lake, Maranbão state, Brazil.}

\begin{abstract}
Serrasalmus aff. brandtii and Pygocentrus nattereri are two species of piranhas, both common in the Viana Lake, which is formed by the flooding waters of the Pindaré River, a tributary of the left bank of the Mearim River. A sample composed of 249 specimens of these species was studied in order to identify diet composition and feeding strategies employed by the two species. The piranhas were captured thrimonthly, during one year from March 1998 to March 1999 using gill and fishing nets of different mesh sizes. The qualitative composition of the diet was analysed through the frequency of occurrence method. The feeding strategies employed by the piranhas were inferred using the graphic method of Costello (1990), modified by Amundsen et al. (1996). The results indicated that fish was the main food item in the stomach contents of the two species, followed by plant material, more in the P. nattereri. Fish and plant material showed different relationships in relation to piranhas's length classes. The graphic analysis of the feeding strategies employed by P. nattereri and S. aff. brandtii suggest a generalist habit, wide width niche with strong participation of the within-phenotype component, which indicated that the majority of individuals of these species use several resources simultaneously. A multivariate analysis of the ecomorphological index indicated that the species are discriminated by swimming ability, water column position and relative prey size.
\end{abstract}

\section{KEYWORDS}

feeding strategies, Serrasalminae, ecomorphology, tropical freshwater.

\footnotetext{
${ }^{1}$ Departamento de Oceanografia e Limnologia/UFMA. Campus do Bacanga. Av. dos Portugueses, s/n. CEP 65085-580, São Luís - MA. E-mail: piorski@ ufma.br
} 


\section{INTRODUÇÃO}

O Lago de Viana está localizado dentro da Área de Proteção Ambiental da Baixada Maranhense. É um lago de várzea, formado pela inundação das águas do rio Pindaré, afluente da margem esquerda do rio Mearim. Na estação chuvosa (janeiro a junho), rios e lagos perenes extravasam, transformando-se em extensos lagos rasos. No período de águas baixas (junho a dezembro), ocorre a concentração de material orgânico na água e aumento da floração de algas e da produtividade de peixes. Segundo Costa Neto (1996), a piscosidade dessa região provavelmente é devida à fertilização dos campos graças aos excrementos de gado durante seu pastoreio de "verão".

No Lago de Viana, duas espécies de piranhas são comuns, Pygocentrus nattereri e Serrasalmus aff. brandtii. A primeira espécie está amplamente distribuída pelos rios da região central e nordeste da América do Sul, a leste dos Andes, incluindo rios costeiros das Guianas e Brasil (Fink, 1993). Serrasalmus brandtii é citada, apenas, para o rio das Velhas (Fowler, 1950) e região de Três Marias (Britski et al., 1988).

Pygocentrus nattereri é caracterizada por apresentar perfil dorsal convexo, focinho curto e arredondado com mandíbula volumosa e prognata. O corpo tem um perfil geral arredondado e padrão de colorido cinza-prateado, com o dorso mais escuro e região antero-ventral alaranjada a avermelhada. Serrasalmus aff. brandtii, por sua vez, apresenta perfil dorsal côncavo na região occipital. O corpo é mais alongado e mais baixo do que $P$. nattereri, sendo o padrão de colorido cinzaprateado com manchas escuras dispersas pelos flancos. Espécies fenotipicamente muito próximas e com adaptações semelhantes para uso dos recursos fornecidos pelo ambiente apresentam forte potencial para competição, a qual pode produzir dois padrões básicos que permitem a convivência (Wootton, 1990): a segregação seletiva e a segregação interativa. Entretanto, em ambientes tropicais a competição pode ser reduzida, devido à flexibilidade alimentar apresentada pela maioria das espécies (Araújo-Lima et al., 1995; Kido, 2001), que pode estar relacionada com a disponibilidade de recursos no ambiente (Lowe-McConnell, 1979; Winemiller, 1989; Jepsen t al., 1997; Winemiller \& Jepsen, 1998) e/ou com as características fenotípicas que possibilitam o uso do recurso disponível (Wainwright \& Richard, 1995; Winemiller et al., 1995; Labropoulou \& Eleftheriou, 1997; Bouton et al., 1999; Mittelbach et al., 1999; Bellwood \& Wainwright, 2001).

Neste trabalho os hábitos alimentares de $P$. nattereri e $S$. aff. brandtii foram estudados com o objetivo de identificar a composição da dieta de cada uma das espécies, bem como as estratégias desenvolvidas para evitar ou reduzir a sobreposição ecológica.

\section{MATERIAL E MÉTODOS}

Os exemplares de piranhas foram coletados trimestralmente entre Março de 1998 e Março de 1999 no lago de Viana (03¹3'43"S, $\left.44^{\circ} 59^{\prime} 96^{\prime \prime O}\right)$, APA da Baixada Maranhense. Durante as coletas foram utilizadas redes de emalhar de diferentes tamanhos e tarrafas, sendo as piranhas capturadas, principalmente, com o primeiro tipo de apetrecho de pesca. Quatro pontos de amostragem foram estabelecidos: Igarapé do Engenho, Ponta das Pedrinhas, Ilha do Carará e Pano Grosso. Em cada um desses pontos foi empregado um esforço de pesca de aproximadamente seis horas contínuas em cada período de coleta. O material coletado foi fixado em formalina $10 \%$ e transportado para o laboratório. Posteriormente, foram transferidos para álcool $70 \%$ e depositados na Coleção de Peixes do Departamento de Oceanografia e Limnologia da Universidade Federal do Maranhão.

\section{Alimentação}

Os hábitos alimentares de $P$. nattereri e $S$. aff. brandtii foram estudados através da análise do conteúdo estomacal. O comprimento padrão (CP) de cada indivíduo foi obtido com paquímetro de precisão de $0.05 \mathrm{~mm}$.

O trato digestivo foi extraído e seu conteúdo exposto em placas de Petri para identificação dos itens alimentares sob estereomicroscópio e microscópio óptico. A relação entre o comprimento do intestino e a dieta foi estudada através do quociente intestinal (QI), resultado da divisão entre o comprimento do intestino e o comprimento padrão do peixe (Zavala-Camin, 1996). O QI médio de cada espécie foi comparado através do teste t de Student.

Os itens alimentares identificados foram agrupados em cinco classes: Peixes, Ephemeroptera, Odonata, Nematoda e Vegetais. Material inorgânico, identificado como material do substrato foi agrupado na categoria Sedimentos, enquanto na categoria Outros foram incluídos materiais de origem desconhecida. A composição qualitativa da dieta foi analisada por meio do método de frequência de ocorrência (Hyslop, 1980), medida pela quantidade de vezes que um determinado tipo de alimento ocorre em relação à soma das ocorrências de todos os ítens, expressa em porcentagem.

A variação da composição da dieta em função do tamanho das presas foi estudada considerando-se seis classes de comprimento: classe $1(\mathrm{CP} \leqslant 50 \mathrm{~mm})$, classe $2(51 \mathrm{~mm} \leqslant \mathrm{CP} \leqslant 70 \mathrm{~mm})$, classe 3 $(71 \mathrm{~mm} \leqslant \mathrm{CP} \leqslant 90 \mathrm{~mm})$, classe $4(91 \mathrm{~mm} \leqslant \mathrm{CP} \leqslant 110 \mathrm{~mm})$, classe 5 $(111 \mathrm{~mm} \leqslant \mathrm{CP} \leqslant 130 \mathrm{~mm})$ e classe $6(\mathrm{CP}>130 \mathrm{~mm})$.

O Índice de Sobreposição Proporcional (Schoener, 1968) foi calculado para avaliar o grau de sobreposição na dieta entre as duas espécies piranhas, utilizando-se a fórmula

$$
R_{o}=1-\frac{1}{2}\left(\sum\left|P_{i j}-P_{i k}\right|\right)
$$

onde: $R_{o}$ é o valor de sobreposição; $P_{i j}$ e $P_{i k}$ são as composições proporcionais do item alimentar $i$ para as espécies $j$ e $k$, respectivamente. O valor de sobreposição será 0 , quando as espécies apresentarem dietas completamente distintas e 1 quando as espécies apresentarem sobreposição total dos itens alimentares. 


\section{ACTA \\ AMAZONICA}

ALIMENTAÇÃO E ECOMORFOLOGIA DE DUAS ESPÉCIES DE PIRANHAS (CHARACIFORMES: CHARACIDAE) DO LAGO DE VIANA, ESTADO DO MARANHÃO, BRASIL.
As estratégias alimentares das duas espécies piranhas foram analisadas por meio do método gráfico de Costello (1990), modificado por Amundsen et al. (1996). Por este método, informações sobre a ecologia alimentar de predadores são obtidas através da relação gráfica entre a abundância presaespecífica (Pi) e sua frequência de ocorrência (Fi). A abundância presa-específica foi calculada pela seguinte fórmula:

$$
\% \mathrm{Pi}=(\Sigma \mathrm{Si} / \Sigma \mathrm{Sti}) \times 100
$$

onde,

$\mathrm{Si}=$ número de estômagos que contêm apenas a presa $i$.

$\mathrm{Sti}=$ total de estômagos em que a presa $i$ ocorre .

A frequência de ocorrência foi obtida pela equação:

$$
\% \mathrm{Fi}=(\mathrm{Ni} / \mathrm{N}) \times 100
$$

onde,

$\mathrm{Ni}=$ número de predadores com a presa $i$ no estômago

$\mathrm{N}=$ total de predadores com conteúdo estomacal.

\section{Ecomorfologia}

Afim de identificar as diferenças morfológicas associadas com o habitat das espécies, foram obtidas 14 medidas morfométricas em 30 exemplares de $P$. nattereri e 80 de $S$. aff. brandtii, descritas abaixo de acordo com os trabalhos de Watson \& Balon (1984), Balonet al. (1986) e Uieda (1995): 1) Comprimento padrão: distância da parte mais anterior da cabeça até o fim da coluna vertebral; 2) Altura máxima do corpo: maior distância vertical do corpo; 3) Largura do corpo: distância entre as bases anteriores das nadadeiras peitorais; 4) Comprimento do pedúnculo caudal: distância da base posterior da anal até o final da coluna vertebral; 5) Altura do pedúnculo caudal: medida em sua porção mediana; 6) Largura do pedúnculo caudal: medida em sua porção mediana; 7) Comprimento máximo da nadadeira peitoral: distância da base ao ponto mais distal da nadadeira; 8) Largura máxima da nadadeira peitoral: medida no ponto de maior largura da nadadeira; 9) Distância máxima vertical da nadadeira caudal: distância máxima entre os raios dos lobos superior e inferior, quando totalmente estendidos; 10) Comprimento máximo da nadadeira caudal: medida entre a extremidade da coluna vertebral e a extremidade do maior raio da nadadeira; 11) Altura da cabeça abaixo do meio do olho: distância vertical entre a porção mediana da órbita e a região ventral da cabeça; 12) Comprimento da cabeça: distância da ponta do focinho até a margem posterior do opérculo; 13) Largura da boca: distância entre as porções laterais da boca, quando totalmente aberta; 14) Altura da boca: distância entre as mandíbulas superior e inferior, com a boca totalmente aberta.

A partir desse conjunto de medidas, foram obtidos nove índices, representando atributos morfológicos que refletem a ecologia de peixes fluviais (Gatz, 1979). Os métodos de obtenção e as interpretações de cada atributo são apresentados abaixo, de acordo com Gatz (1979) e Mahon (1984).

1) Índice de compressão (IC): razão entre a altura máxima do corpo e a largura máxima do corpo. Altos valores indicam forte compressão lateral do peixe, comum em peixes que ocupam habitats comágua em baixa velocidade; 2) Altura relativa do corpo (ARC): razão entre a altura máxima do corpo e o comprimento padrão. Está inversamente relacionado à velocidade desenvolvida pelo animal e diretamente relacionado à capacidade de fazer giros verticais; 3) Comprimento relativo do pedúnculo caudal (CRPC): razão entre o comprimento do pedúnculo caudal e o comprimento padrão. Está relacionado com a habilidade natatória; 4) Índice de compressão do pedúnculo caudal (ICPC): razão entre a altura e a largura do pedúnculo caudal. Este índice relaciona-se inversamente com a velocidade de natação e diretamente com a capacidade de realizar manobras; 5) Configuração da nadadeira peitoral (CNP): razão entre o comprimento máximo e a largura máxima da nadadeira peitoral. Está relacionada com a intensidade de natação; 6) Comprimento relativo da cabeça (CRC): razão entre o comprimento da cabeça e o comprimento padrão. Está relacionado com o tamanho da presa consumida; 7) Posição relativa do olho (PRO): razão entre a altura da cabeça abaixo do meio do olho e o comprimento padrão. Indica a posição do animal na coluna d'água; 8) Largura relativa da boca (LRB): razão entre a largura da boca e o comprimento padrão; 9) Altura relativa da boca (ARB): razão entre a altura da boca e o comprimento padrão. Os dois últimos índices relacionam-se com o tamanho das presas.

Os atributos morfológicos foram organizados em uma matriz de correlação e submetidos a uma análise de componentes principais (ACP), utilizando a rotina Factor Analysis do pacote STATISTICA (StatSoft, 2001), a fim de identificar padrões ecomorfológicos que descrevem o conjunto de dados analisados (Monteiro \& Reis, 1999). Seguindo a recomendação de Watson \& Balon (1984) foi realizado uma rotação ortogonal através da opção VARIMAX. Este procedimento maximiza a correlação com outros fatores. Para evitar superfatorização, apenas os fatores com autovalores maiores que 1,0 foram considerados significantes.

\section{RESULTADOS}

\section{Alimentação}

Foram analisados 150 exemplares de Pygocentrus nattereri com comprimento padrão variando de 48 a $150 \mathrm{~mm}$ e 99 exemplares de Serrasalmus aff. brandtii com variação de 41 a $146 \mathrm{~mm}$ CP. Para P. nattereri foi registrado Quociente Intestinal médio de 1.033 , enquanto que para $S$. aff. brandtii o QI médio foi de $0.771(\mathrm{t}=13.09155, \mathrm{p}<0.005)$.

Peixes foi o item presente com maior frequência no conteúdo estomacal de $P$. nattereri e $S$. aff. brandtii (Fig. 1).

O segundo item mais frequente foi vegetais, seguido de Ephemeroptera, Odonata e Nematoda (Fig. 1). A análise da importância das categorias alimentares, através do diagrama de Costello (Fig. 2), revelou que peixes foi o item dominante na dieta das duas espécies, sendo consumido por todos os indivíduos da população. Vegetais foi um recurso explorado pela maior parte da população, enquanto que os demais itens podem ser considerados componentes ocasionais da dieta. 


\section{ACTA AMAZONICA}

ALIMENTAÇÃO E ECOMORFOLOGIA DE DUAS ESPÉCIES DE PIRANHAS

(CHARACIFORMES: CHARACIDAE) DO LAGO DE VIANA, ESTADO DO MARANHÃO, BRASIL.
De acordo com o diagrama de Costello (Fig. 2), P. nattereri e $S$. aff. brandtii adotam a estratégia generalista, apresentando ampla largura de nicho com relativa importância do componente intra-fenótipo.
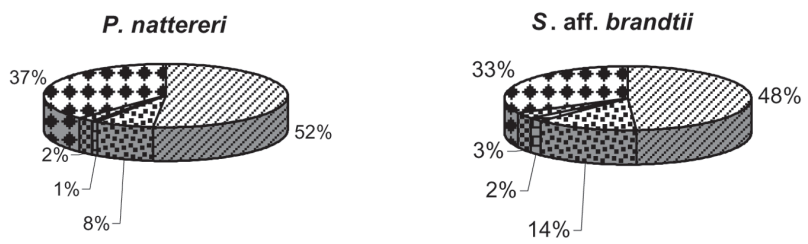

\section{$\checkmark$ Peixes $\quad$ Ephemeroptera $\quad$ OOdonata Nematoda Vegetais}

Figura 1 - Composição percentual da dieta de $P$. nattereri $(\mathrm{n}=$ 150) e $S$. aff. brandtii $(\mathrm{n}=99)$ no Lago de Viana entre abril de 1998 e fevereiro de 1999.
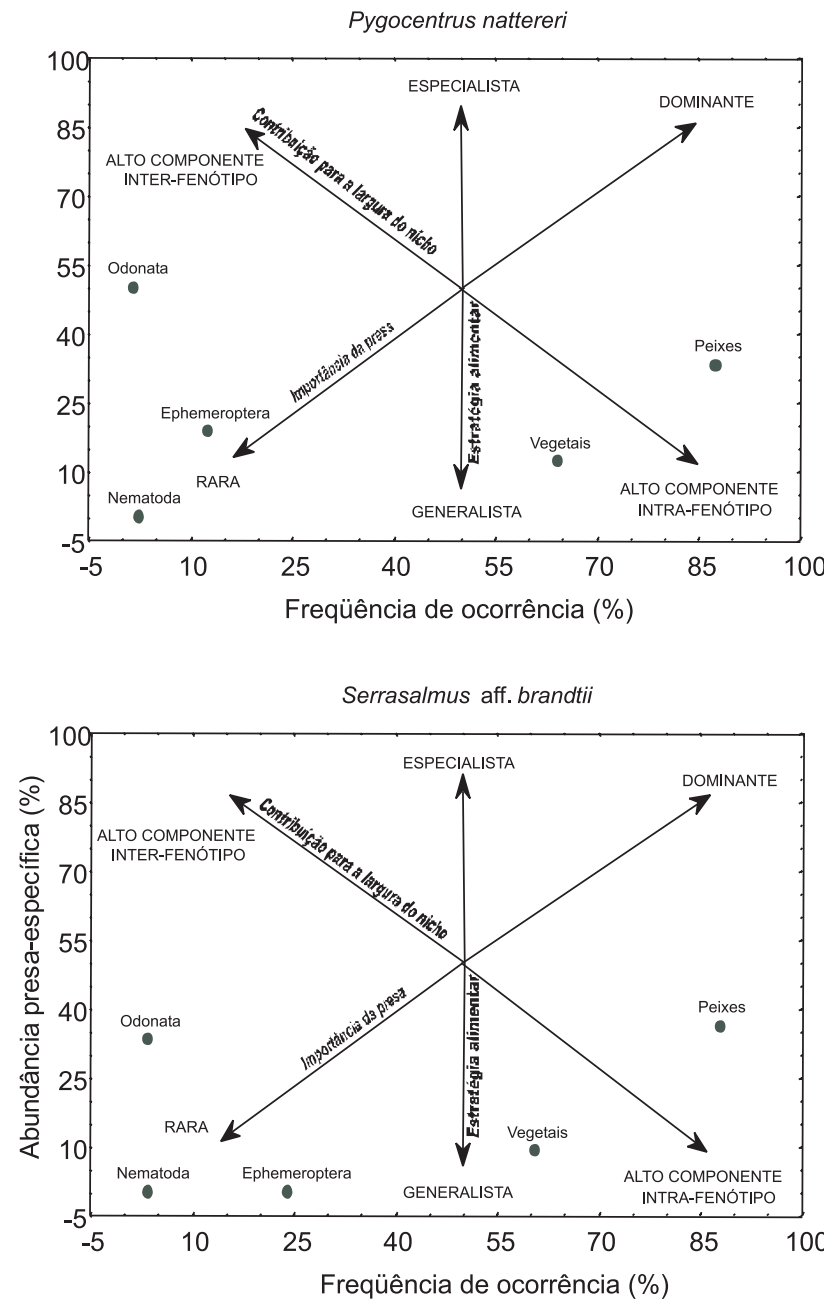

Figura 2 - Diagrama de Costello mostrando a relação gráfica entre a frequência de ocorrência e a abundância presa-específica na dieta de $P$. nattereri $(\mathrm{n}=150)$ e $S$. aff. brandtii $(\mathrm{n}=99)$.
A análise temporal dos dados indicou que os ítens peixes, efemerópteros e vegetais foram encontrados nos estômagos de $S$. aff. brandtii em todos os meses de amostragem (Fig. 3). Odonata esteve presente apenas em abril/98, enquanto Nematoda ocorreu nos meses de agosto e novembro de 1998. Nesta espécie, o item peixes sempre ocorreu em mais da metade dos estômagos analisados, sendo abril/98 o mês com menor frequência e novembro/98 o mês com maior frequência. $\mathrm{O}$ item vegetais, por outro lado, foi menos frequente em novembro/98 quando ocorreu em apenas $14.29 \%$ dos estômagos analisados. As maiores frequências para este item foram obtidas nos meses de agosto/98 e fevereiro/99 (Fig. 3).

Nos estômagos de $P$. nattereri apenas duas categorias de alimento (peixes e vegetais) foram observadas ao longo de todos os meses de amostragem (Fig. 3). Ephemeroptera e Nematoda ocorreram apenas em agosto/98, ao passo que Odonata ocorreu em abril/98 e novembro/98. Da mesma forma que $S$. aff. brandtii, a análise do conteúdo estomacal de $P$. nattereri revelou que o item peixes apresentou frequência de ocorrência acima de $60 \%$ em todos os períodos, embora estas frequências tenham sido mais baixas do que aquelas registradas para S. aff. brandtii (Fig. 3).

A composição da dieta nas diferentes classes de comprimento de $S$. aff. brandtii mostra, essencialmente, a mesma estrutura para a composição geral da dieta da espécie (Fig. 4). Não houve relação entre a proporção de peixes na dieta e o tamanho dos exemplares de $S$. aff. brandtii $\left(\mathrm{R}^{2}=\right.$ $0,15, b=-0,39, p=0,44)$. Por outro lado, exemplares maiores consumiram mais material vegetal $\left(\mathrm{R}^{2}=0,73, \mathrm{~b}=0,86, \mathrm{p}=\right.$ 0,03 ) (Fig. 4). Efemerópteros foram consumidos por exemplares de todas as classes de comprimento.

Para P. nattereri o consumo de vegetais parece ocorrer na mesma proporção que o de peixes nos indivíduos das três primeiras classes de comprimento (Fig. 4). Entretanto, esta observação pode ter sido influenciada pelo pequeno número de indivíduos associados a estas classes de tamanho. Em indivíduos maiores a dieta é composta por uma proporção maior de peixes em relação aos demais itens, sem apresentar nenhuma tendência de redução ou aumento no consumo de peixes $\left(R^{2}=0,406, b=0,637, p=0,1736\right)$ e vegetais $\left(R^{2}=\right.$ $0,3089, b=-0,56, p=0,2522)$ em função do tamanho. Ao contrário de $S$. aff. brandtii, efemerópteros foram consumidos apenas pelos indivíduos maiores.

Para o Índice de Sobreposição Proporcional foi obtido um valor de 0,85 , indicando alta taxa de sobreposição entre as dietas das duas espécies de piranhas estudadas.

\section{Ecomorfologia}

A análise de Componentes Principais, aplicada sobre a matriz combinada dos atributos morfológicos de $P$. nattereri $\mathrm{e}$ $S$. aff. brandtii, permitiu a identificação dos caracteres ecomorfológicos que diferenciam as duas espécies através dos dois primeiros componentes principais, onde $64 \%$ da variação foi acumulada (Tab. 1, Fig. 5). A maior parte da variação (43,4\%) 

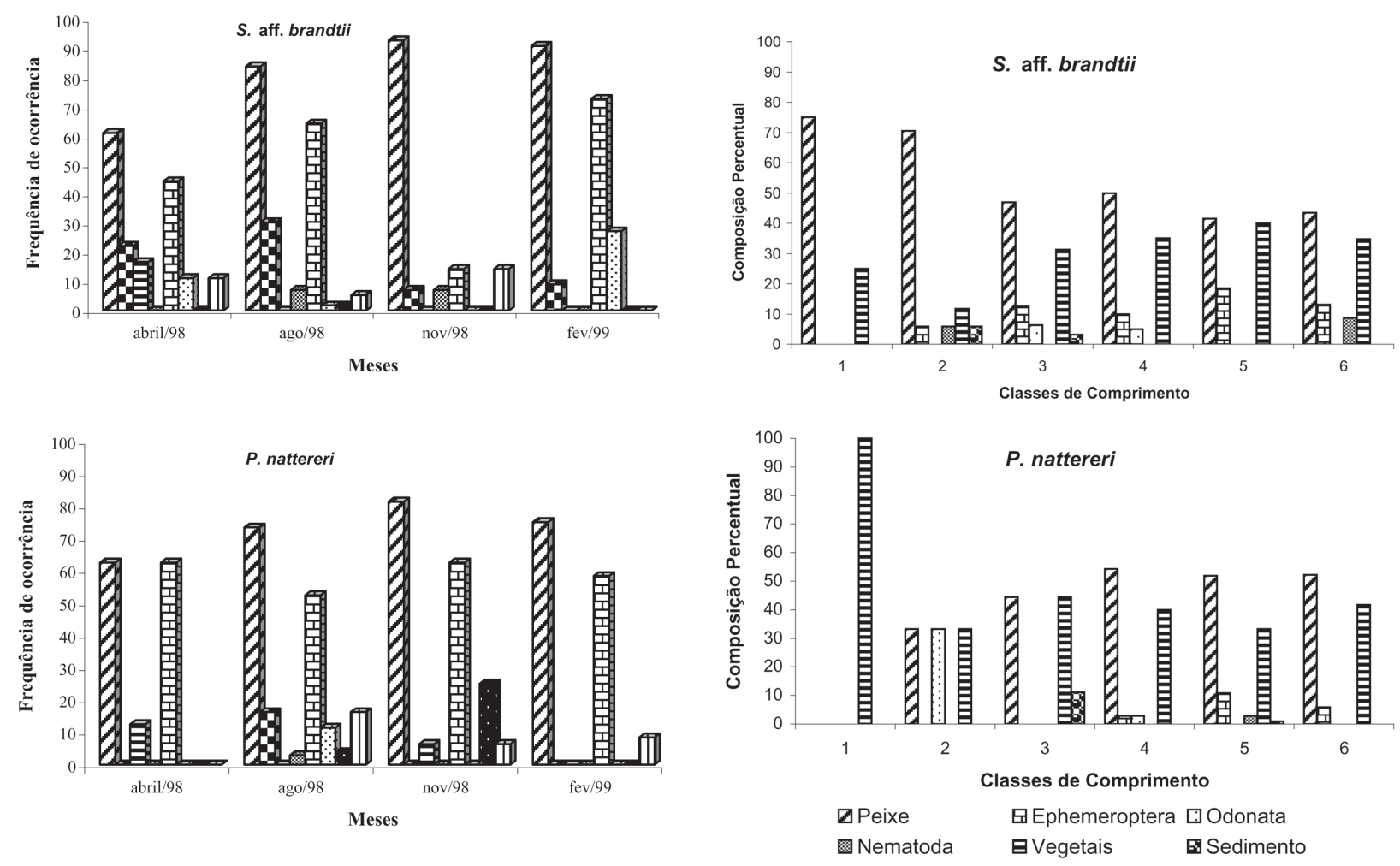

\begin{tabular}{|c|c|c|}
\hline $\begin{array}{l}\square \text { Peixes } \\
\text { Nematoda } \\
\text { Outros }\end{array}$ & $\begin{array}{l}\text { 口Ephemeroptera } \\
\text { 可egetais } \\
\text { Estômago vazio }\end{array}$ & $\begin{array}{l}\text { EOdonata } \\
\text { Sedimentos }\end{array}$ \\
\hline
\end{tabular}

Figura 3 - Variação temporal na frequência de ocorrência dos ítens alimentares nos estômagos de $S$. aff. brandtii $(\mathrm{n}=99) \mathrm{e}$ P. nattereri $(\mathrm{n}=150)$ do Lago de Viana entre abril de $1998 \mathrm{e}$ fevereiro de 1999.

foi explicada pelo primeiro componente principal, indicando que as variáveis Altura Relativa do Corpo, Comprimento Relativo da Cabeça, Posição Relativa do Olho, Largura Relativa da Boca e Altura Relativa da Boca são maiores em $P$. nattereri (Tab. 1). A combinação destas variáveis, ao longo deste componente, permitiu a identificação de três fatores importantes para a discriminação das espécies: agilidade natatória, tamanho potencial da presa consumida e posição na coluna d'água (Fig. 5).

O segundo componente principal explicou $21 \%$ da variação e foi fortemente influenciado pelo Índice de Compressão Lateral, Índice de Compressão do Pedúnculo Caudal e Configuração da Nadadeira Peitoral, que apresentaram maiores valores em $S$. aff. brandtii.

\section{DISCUSSÃO}

Piranhas são peixes com estrutura dentária adaptada para arrancar pedaços de suas vítimas, que são engolidas sem mastigar. Estudos recentes têm demonstrado que diversas espécies de piranhas apresentam grande variedade de hábitos alimentares (Goulding et al., 1988; Sazima \& Machado, 1990).

Figura 4 - Variação da composição percentual dos diferentes ítens alimentares presentes no conteúdo estomacal de $S$. aff. brandtii $(\mathrm{n}=99)$ e $P$. nattereri $(\mathrm{n}=150)$ por classe de tamanho. [Classe $1(\mathrm{CP} \leqslant 50 \mathrm{~mm})$, classe $2(51 \mathrm{~mm} \leqslant \mathrm{CP} \leqslant 70 \mathrm{~mm})$, classe $3(71 \mathrm{~mm} \leqslant \mathrm{CP} \leqslant 90 \mathrm{~mm})$, classe $4(91 \mathrm{~mm} \leqslant \mathrm{CP} \leqslant 110 \mathrm{~mm})$, classe $5(111 \mathrm{~mm} \leqslant \mathrm{CP} \leqslant 130 \mathrm{~mm})$ e classe $6(\mathrm{CP}>130 \mathrm{~mm})]$

Luiz et al. (1998) observaram que a espécie Serrasalmus marginatus tem como principal recurso alimentar peixes, seguido de restos de vegetais. Com base nesses dados, os autores a classificaram como piscívora. Em estômagos de outra espécie do mesmo gênero, S. altuvei, Leão et al. (1991) observaram peixes inteiros, matéria vegetal, escamas, insetos adultos, ovos e larvas de insetos, classificando-a como onívora com tendência à piscivoria.

Da mesma forma que Serrasalmus marginatus e S. altuvei, as espécies $S$. aff. brandtii e $P$. nattereri, investigadas neste trabalho, apresentam alimentação diversificada. A tendência à piscivoria é confirmada pelo quociente intestinal. Os valores deste índice são intermediários para aqueles que caracterizam as categorias tróficas de carnívoros e onívoros para ciclídeos da Amazônia (Reis \& Caramaschi, 1999), e acima dos valores obtidos por Barbieri et al. (1994) para as categorias carnívoro generalista e piscívoros, aproximando-se das duas categorias de onívoros identificadas por estes autores para peixes de uma localidade do sudeste do Brasil. Do mesmo modo, a análise gráfica das estratégias alimentares empregadas por $P$. nattereri $\mathrm{e} S$. aff. brandtii sugerem um hábito generalista $\mathrm{e}$ ampla largura de nicho, com relativa participação do 
Tabela 1 - Cargas dos atributos ecomorfológicos nos dois primeiros componentes principais. Os atributos mais importantes para definição dos fatores que discriminam as espécies $(n=110)$ estão marcados com asteriscos.

\begin{tabular}{lcc}
\hline \hline Atributo Ecomorfológico & CP1 & CP2 \\
\hline Índice de Compressão Lateral & $-0,416$ & $0,712^{*}$ \\
Altura Relativa do Corpo & $0,816^{*}$ & 0,111 \\
Comp. Relativo do Pedúnculo Caudal & 0,620 & $-0,283$ \\
Índice de Compressão do Ped. Caudal & 0,096 & $0,692^{*}$ \\
Configuração da Nadadeira Peitoral & $-0,232$ & $0,767^{*}$ \\
Comprimento Relativo da Cabeça & $0,834^{*}$ & $-0,115$ \\
Posição Relativa do Olho & $0,835^{*}$ & $-0,332$ \\
Largura Relativa da Boca & $0,897^{*}$ & $-0,268$ \\
Altura Relativa da Boca & $0,647^{*}$ & $-0,120$ \\
Autovalor & 3,903 & 1,876 \\
Variância explicada & 0,434 & 0,208 \\
\hline \hline
\end{tabular}

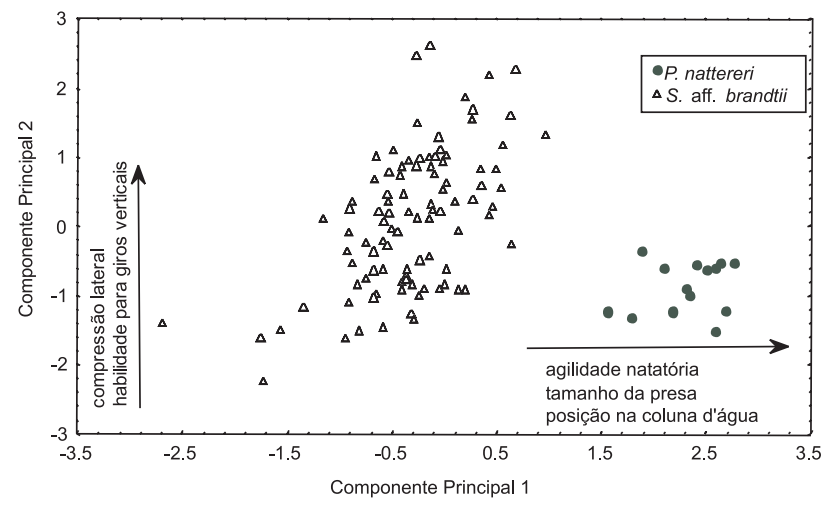

Figura 5 - Distribuição dos escores individuais de $S$. aff. brandtii $(\mathrm{n}=80)$ e $P$. nattereri $(\mathrm{n}=30)$ no espaço dos dois primeiros componentes principais.

componente intra-fenótipo, indicando que a maioria dos indivíduos destas espécies utilizam vários tipos de recursos simultaneamente (Wootton, 1990).

No Lago de Viana $S$. aff. brandtii apresenta um consumo de matéria vegetal elevado, contrapondo-se aos dados obtidos para esta espécie nas lagoas marginais do rio São Francisco (Pompeu, 1999). Naquelas lagoas, matéria vegetal contribuiu com menos de $10 \%$ na composição da dieta de S. brandtii.

Pompeu (1999) também observou variações na dieta associadas com o crescimento, onde o consumo de insetos aquáticos nos indivíduos menores é completamente substituído por uma dieta piscívora nos adultos. Do mesmo modo, Bistoni \& Haro (1995) detectaram variações ontogenéticas na alimentação de $S$. spilopleura, onde indivíduos jovens com menos de $10 \mathrm{~mm}$ de comprimento total têm suas dietas completadas por larvas de crustáceos e odonata, e acima de $10 \mathrm{~mm}$ alimentam-se mais comumente de peixes. Este não é o caso dos espécimes de $S$. aff. brandtii do
Lago de Viana, em que a piscivoria está presente em todas as classes de tamanho. Ao contrário de modificações drásticas na dieta, nossos resultados indicam que as espécies de piranhas estudadas mantêm a mesma composição da dieta nas diferentes fases do crescimento, ocorrendo apenas variações nas proporções dos itens alimentares. Assim, pelo menos em $S$. aff. brandtii, a piscivoria parece ser mais evidente nos indivíduos com menos de $70 \mathrm{~mm}$ de comprimento, assumindo posteriormente uma dieta muito próxima da onivoria.

Segundo Goulding (1980), frutos e sementes são dois itens bastante necessários à dieta de piranhas. Entretanto, em nenhum estômago das espécies estudadas neste trabalho foram encontrados frutos. Sementes foram observadas ocasionalmente.

Em peixes, a composição da dieta pode apresentar variações sazonais. Estas variações podem ocorrer devido a alterações na disponibilidade de alimentos provocada por mudanças nos habitats disponíveis para forrageamento, mudanças devido a padrões biológicos dos organismos - presa e mudanças provocadas pelas atividades alimentares dos peixes em si (Wooton, 1990). Nas lagoas do rio São Francisco, Pompeu (1999) observou que $S$. aff. brandtii apresentou aumento no consumo de insetos aquáticos durante a estação chuvosa. No presente trabalho, não observamos variações acentuadas no conteúdo estomacal das piranhas considerando-se as estações seca e chuvosa.

A análise do conteúdo estomacal e o valor obtido para o índice de sobreposição revelaram grande semelhança dos hábitos alimentares de $S$. aff. brandtii e $P$. nattereri. A potencial competição entre essas espécies pode ser minimizada pela diversificação alimentar e pelo uso do ambiente. Aespécie $S$. aff. brandtii, por exemplo, parece equacionar sua dieta consumindo proporcionalmente maior variedade de itens, ao passo que $P$. nattereri intensifica sua predação sobre peixe e matéria vegetal.

\section{Ecomorfologia}

A análise combinada dos caracteres ecomorfológicos de $P$. nattereri e $S$. aff. brandtii indicou que estas espécies diferenciam-se por fatores associados com agilidade natatória, tamanho potencial da presa e posição de natação na coluna d'água. Dentre estes, o fator mais importante parece ser o tamanho potencial da presa. Embora não se tenha observado a presença de peixes inteiros no conteúdo estomacal das duas espécies, a atribuição de maiores valores das variáveis Largura Relativa da Boca e Altura Relativa da Boca para P. nattereri sugere maior aptidão para a captura de presas de tamanhos maiores do que seria capaz $S$. aff. brandtii.

Características morfológicas relacionadas ao tamanho da presa capturada representam, de modo geral, uma das principais adaptações desenvolvidas por espécies predadoras simpátricas em resposta à sobreposição na dieta e consequente competição (Huskey \& Turingan, 1998; Piet, 2000; Freire \& Agostinho, 2001; Cussac et al. 2002). Labropoulou \& Eleftheriou (1997), por exemplo, observaram diferenças significantes na 


\section{ACTA \\ AMAZONICA}

ALIMENTAÇÃO E ECOMORFOLOGIA DE DUAS ESPÉCIES DE PIRANHAS (CHARACIFORMES: CHARACIDAE) DO LAGO DE VIANA, ESTADO DO MARANHÃO, BRASIL. morfologia do aparelho alimentar entre pares de espécies simpátricas, sugerindo a segregação de nicho trófico entre essas espécies. Hugueny \& Pouilly (1999) identificaram em uma assembléia de peixes de água doce que o hábito piscívoro apresenta correlação positiva com a altura da boca e comprimento padrão, confirmando as idéias de Wainwright \& Richard (1995) de que o tamanho da boca deve exercer um papel importante na variação interespecífica na dieta dentro das comunidades de peixes.

É possível que uma das alternativas encontradas pelas espécies para possibilitar a convivência foi o desenvolvimento de adaptações que permitem a predação de presas grandes ou pequenas e lentas ou rápidas. Assim, $P$. nattereri apresenta um fenótipo que lhe permite a captura de presas grandes e que se movimentam mais rapidamente do que as presas que compõem a dieta de $S$. aff. brandtii. Consequentemente, uma análise mais detalhada da dieta destas espécies pode revelar uma composição diferenciada de presas. No presente trabalho isto não foi possível, pois os peixes observados no conteúdo estomacal eram representados por pedaços que impediam o reconhecimento taxonômico.

A escolha de um determinado tipo de presa, de certa forma, está relacionada às características morfológicas do aparelho alimentar do predador (Labropoulou \& Eleftheriou, 1997). Entretanto, fenótipos que indicam um hábito especialista podem estar combinados com atividades alimentares generalistas, configurando o que é conhecido como paradoxo de Liem (Bouton et al., 1998). Como apresentado anteriormente, parece haver maior variabilidade na dieta de $S$. aff. brandtii do que em $P$. nattereri. A não correspondência entre fenótipo e atividade alimentar, tal como previsto pelo paradoxo de Liem, pode ser vantajoso para o especialista, pois ele pode aumentar o espectro de ítens-presa em sua dieta, explorando tanto recursos de fácil obtenção quanto de difícil obtenção, ao passo que o consumidor fenotipicamente generalista não apresenta habilidade especializada para a exploração de recursos de difícil obtenção (Bouton et al., 1998). Por outro lado, S. aff. brandtii e $P$. nattereri são duas espécies fenotipicamente especializadas, podendo-se questionar a razão pela qual $S$. aff. brandtii consome maior quantidade de vegetais e invertebrados do que a outra espécie. É provável que a combinação de elevado índice de compressão com elevado valor da configuração da nadadeira peitoral, tal como evidenciado pela ACP, tenha munido a espécie das condições necessárias para exploração de outros recursos alimentares. Se esta hipótese for correta, a solução do paradoxo de Liem (Robinson \& Wilson, 1998) não depende apenas da presença/ausência de recursos fáceis no ambiente e que possam, dessa forma, serem utilizados por espécies fenotipicamente especializadas, mas também, das demais características morfológicas do animal que conferem pré-adaptações para a exploração do recurso.

Hugueny \& Pouilly (1999), discutindo a validade de estudos ecomorfológicos, sugerem que a disponibilidade de alimento possa ser um dos fatores que impossibilitam o estabelecimento de relações entre dieta e morfologia se o estudo está restrito a espécies que pertencem à mesma categoria trófica. Entretanto, mesmo nestes casos, as características ecomorfológicas das espécies envolvidas no estudo podem fornecer informações úteis sobre a partilha do habitat pelas mesmas. Isto se aplica ao presente trabalho, onde as espécies estudadas pertencem à mesma categoria trófica, mas podem ser diferenciadas com base em atributos ecomorfológicos, os quais sugerem diferenças no tamanho da presa capturada, diferenças no método de obtenção de alimento e na diversidade de recursos explorados.

\section{BIBLIOGRAFIA CITADA}

Amundsen, P.A.; Gabler, H.M.; Staldvik, F.J. 1996. A new approach to graphical analysis of feeding strategy from stomach contents data - modification of the Costello (1990) method. Journal of Fish Biology, 48(4): 607-614.

Araujo-Lima, C.A.R.M.; Agostinho, A.A.; Fabré, N.N. 1995. Trophic aspects of fish communities in brazilian rivers and reservoirs. In: Tundisi, J.G.; Bicudo, C.E.M.; Matsumura-Tundisi, T. (Eds). Limnology in Brazil. Rio de Janeiro: ABC/ABL. 105-136 p.

Ball, J.N. 1961. On the food of the brown trout of Llyn Tegid. Proceedings of the Zoological Society of London, 137: 599-622.

Balon, E.K.; Crawford, S.S.; Lelek, A. 1986. Fish communities of the upper Danube River (Germany, Austria) prior to the new Rhein-Main-Donan connection. Environmental Biology of Fishes, 15: 243-271.

Barbieri, G.; Peret, A.C.; Verani, J.R. 1994. Notas sobre a adaptação do trato digestivo ao regime alimentar em espécies de peixes da região de São Carlos (SP). I. Quociente Intestinal. Revista Brasileira de Biologia, 54: 63-69.

Bellwood, D.R.; Wainwright, P.C. 2001. Locomotion in labrid fishes: implications for habitat use and cross-shelf biogeography on the Great Barrier Reef. Coral Reefs, 20: 139-150.

Bistoni, M.A.; Haro, J.G. 1995. Habitos alimentarios de Serrasalmus spilopleura (Pisces, Serrasalmidae) en los bañados del rio dulce (Córdoba, Argentina). Revista Brasileira de Biologia, 55: 847-833.

Bouton, N.; Os, N. van; Witte, F. 1998. Feeding performance of Lake Victoria rock cichlids: testing predictions from morphology. Journal of Fish Biology, 53: 118-127.

Bouton, N.; Witte, F.; van Alphen, J.J.M.; Schenk, A.; Seehausen, O. 1999. Local adaptations in populations of rock-dwelling haplochromines (Pisces: Cichlidae) from southern Lake Victoria. Proceedings of the Royal Society of London, series B, Biological Sciences, 266: 235-360.

Britski, H.A.; Sato, Y.; Rosa, A.B.S. 1988. Manual de identificação de peixes da região de Três Marias. CODEVASF, 3 ED. Belo Horizonte, $115 \mathrm{p}$.

Costa Neto, J.P. 1996. Maranhão: um Estado que precisa ser criado para a cidadania. Desenvolvimento \& Cidadania, 18: 7-11.

Costello, M.J. 1990. Predator feeding strategy and prey importance: a new graphical analysis. Journal of Fish Biology, 36: 261-263.

Cussac, E.V.; Ruzzante, D.; Nalde, S.; Macchi, P.J.; Ojeda, V.; Alonso, M.F.; Denegri, M.A. 2002. Body shape variation of three species of Percichthys in relation to their coexistence in the Limay River basin, in northern Patagonia. Environmental Biology of Fishes, 65: 37-45. 
Fink, W.L. 1993. Revision of the piranha genus Pygocentrus (Teleostei, Characiformes) Copeia, 1993: 665-687.

Fowler, H.W. 1950. Os peixes de água doce do Brasil. Arquivos de Zoologia, VI (2를 entrega): p 380.

Freire, A.G.; Agostinho, A.A. 2001. Ecomorfologia de oito espécies dominantes da ictiofauna do reservatório de Itaipu (Paraná/Brasil). Acta Limnologia Brasiliensia, 13: 1-9.

Gatz Jr., A.J. 1979. Ecological morphology of freshwater stream fishes. Tulane Studies in Zoology and Botany, 21: 91-124.

Goulding, M. 1980. The fishes and the forest exploration in Amazonian natural bistory. Berkeley: University of California Press. 280pp.

Goulding, M.; Carvalho, M.L.; Ferreira, E.G. 1988. Rio Negro: rich life in poor water. SBC Academic Publishing. The Hague, 200pp.

Hugueny, B.; Pouilly, M. 1999. Morphological correlates of diet in an assemblage of West African freshwater fishes. Journal of Fish Biology, 54: 1310-1325.

Huskey, S.H.; Turingan, R.G. 1998. Variation in prey-resource utilization and oral jaw gape between two populations of largemouth bass, Micropterus salmoides. Environmental Biology of Fishes, 51: 67-86.

Hyslop, E.J. 1980. Stomach contents analysis - a review of methods and their application. Journal of Fish Biology, 17: 411-29.

Jepsen, D.B.; Winemiller, K.O.; Taphorn, D.C. 1997. Temporal patterns of resource partitioning among Cichla species in a Venezuelan blackwater river. Journal of Fish Biology, 51: 1085-1108.

Kido, M.C. 2001. Food relations between coexisting native Hawaiian stream fishes. Environmental Biology of Fishes, 61: 185-194.

Labropoulou, M.; Eleftheriou, A. 1997. The foraging ecology of two pairs of congeneric demersal fish species: importance of morphological characteristics in prey selection. Journal of Fish Biology, 50: 324-340.

Leão, E.L.M.; Leite,R.G.; Chaves, P.T.C.; Ferraz, E. 1991. Aspectos da reprodução, alimentação e parasitofauna de uma espécie rara de piranha, Serrasalmus altuvei Ramírez, 1965 (Pisces, Serrasalmidae) do baixo Rio Negro. Revista Brasileira de Biologia, 51: 545-553.

Liem, K.F. 1980. Aquisition of energy by teleosts: adaptive mechanisms and evolutionary patterns. In: Ali, M.A. (Ed). Environmental Physiology of Fishes. New York: Plenum Publishing Corporation. 299-334 pp.

Lowe-McConnell, R.H. 1979. Ecological aspects of seasonality in fishes of tropical waters. Symposia of the Zoological Society of London, 44: 219-241.

Luiz, E.A.; Agostinho, A.A.; Gomes, L.C.; Hann, N.S. 1998. Ecologia trófica de peixes em dois riachos da bacia do Rio Paraná. Revista Brasileira da Biologia, 58: 273-285.

Mahon, R. 1984. Divergent structure in fish taxocenes of North temperate streams. Can. J. Fish. Aquat. Sci., 41: 330-350.
Mittelbach, G.G.; Osenberg, C.W.; Wainwright, P.C. 1999. Variation in feeding morphology between pumpkinseed populations: phenotypic plasticity or evolution? Evolutionary Ecology Research, 1: 111-128.

Monteiro, L.R.; Reis, S.F. 1999. Princípios de morfometria geométrica. Ribeirão Preto: Holos. 188p.

Piet, G.J. 2000. Ecomorphology of a size-structured tropical freshwater fish community. Environmental Biology of Fishes, 59: 191-198.

Pompeu, P.S. 1999. Dieta da pirambeba, Serrasalmus brandtii Reinhardt (Teleostei, Characidae), em quatro lagoas marginais do Rio São Francisco, Brasil. Revista Brasileira de Zoologia, 16 (supl 2): 19-26.

Reis, R.A.; Caramashi, E.P. 1999. Feeding habitats of nine cichlids found in Batata lake (Porto Trombetas, PA, Brazil). In: Val, A.L.; Almeida-Val, V.M.F. (Eds). Biology of tropical fishes. INPA, Manaus, 127-136 p.

Robinson, B.W.; Wilson, D.S. 1998. Optimal foraging, specialization, and a solution to Liem's Paradox. The American Naturalist, 151: 223-235.

Sazima, I.; Machado, F.A. 1990. Underwater observations of piranhas in western Brazil. Environmental Biology of Fishes, 28: 17-31.

Schoener, T.W. 1968. The anolis lizards of Bimini: resource partitioning in a complex fauna. Ecology, 49: 704-726.

StatSoft, Inc. 2001. STATISTICA (data analysis software system), version 6. www.statsoft.com.

Uieda, V.S. 1995. Comunidade de peixes de um riacho litorâneo: composição, habitat e hábitos. Tese de Doutorado, IBUNICAMP. Campinas-SP. 229pp.

Wainwright, P.C.; Richard, B.A. 1995. Predicting patterns of prey use from morphology of fishes. Environmental Biology of Fishes, 44: 97-113.

Watson, D.J.; Balon, E.K. 1984. Ecomorphological analysis of fish taxocenes in rainforest streams of northern Borneo. Journal of Fish Biology, 25: 371-384.

Winemiller, K.O. 1989. Ontogenetic diet shifts and resource partitioning among piscivorous fishes in the Venezuelan llanos. Environmental Biology of Fishes, 26: 177-199.

Winemiller, K.O.; Jepsen, D.B. 1998. Effects of seasonality and fish movement on tropical river food webs. Journal of Fish Biology, 53: 267-296.

Winemiller, K.O.; Kelso-Winemiller, L.C.; Brenkert, A.L. 1995. Ecomorphological diversification and convergence in fluvial cichlid fishes. Environmental Biology of Fishes, 44: 235-261.

Wootton, R.J. 1990. Ecology of teleost fishes. New York: Chapman and Hall. 404 p.

Zavala-Camin, L.A. 1996. Introdução aos estudos sobre alimentação natural de peixes. São Paulo-Maringá: EDUEM. 129pp.

\section{RECEBIDO EM 08/08/2003 \\ ACEITO EM 21/02/2005}

